UCRL-ID-128923

\title{
Antiproton Fast Ignition for Inertial Confinement Fusion
}

\author{
L. John Perkins
}

October 24, 1997

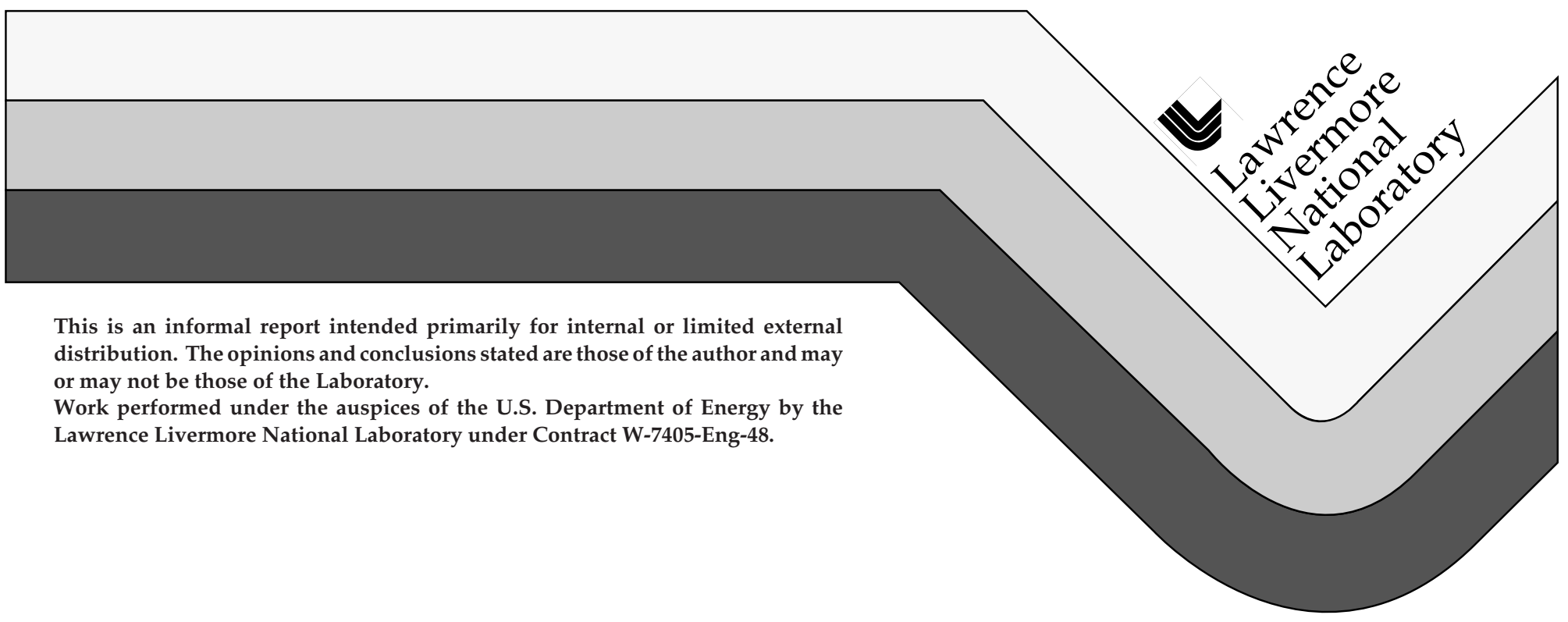




\section{DISCLAIMER}

This document was prepared as an account of work sponsored by an agency of the United States Government. Neither the United States Government nor the University of California nor any of their employees, makes any warranty, express or implied, or assumes any legal liability or responsibility for the accuracy, completeness, or usefulness of any information, apparatus, product, or process disclosed, or represents that its use would not infringe privately owned rights. Reference herein to any specific commercial product, process, or service by trade name, trademark, manufacturer, or otherwise, does not necessarily constitute or imply its endorsement, recommendation, or favoring by the United States Government or the University of California. The views and opinions of authors expressed herein do not necessarily state or reflect those of the United States Government or the University of California, and shall not be used for advertising or product endorsement purposes.

This report has been reproduced directly from the best available copy.

Available to DOE and DOE contractors from the Office of Scientific and Technical Information P.O. Box 62, Oak Ridge, TN 37831

Prices available from (423) 576-8401

Available to the public from the National Technical Information Service

U.S. Department of Commerce 5285 Port Royal Rd., Springfield, VA 22161 


\title{
ANTIPROTON FAST IGNITION FOR INERTIAL CONFINEMENT FUSION*
}

\author{
L. John Perkins \\ Lawrence Livermore National Laboratory
}

October 24, 1997 


\title{
ANTIPROTON FAST IGNITION FOR INERTIAL CONFINEMENT FUSION
}

\author{
L. John Perkins \\ Lawrence Livermore National Laboratory
}

October 24,1997

With $180 \mathrm{MJ} / \mu \mathrm{g}$, antiprotons offer the highest stored energy per unit mass of any known entity. We investigate the use of antiprotons to promote fast ignition in an ICF capsule and seek high gains with only modest compression of the main fuel. Unlike standard fast ignition where the ignition energy is supplied by an energetic, short pulse laser, the energy here is supplied through the ionization energy deposited when antiprotons annihilate at the center of a compressed fuel capsule. In the first of two candidate fast ignition schemes, the antiproton package is delivered by a low energy external ion beam. In the second, "autocatalytic" scheme, the antiprotons are pre-emplaced at the center of the capsule prior to compression. In both schemes, we estimate that $\sim 3 \times 10^{13}$ antiprotons are required to initiate fast ignition in a typical ICF capsule and show that incorporation of a thin, heavy metal shell is desirable to enhance energy deposition in the ignitor zone. In addition to obviating the need for a second energetic fast laser and vulnerable final optics, this scheme would achieve central ignition without reliance on laser channeling through halo plasma or houlrahm debris. However, in addition to the unknowns involve in the storage and manipulation of antiprotons at low energy, the other large uncertainty for the practicality of such a scheme is the ultimate efficiency of antiproton production in an external, optimized facility. Our estimates suggest that the wallplug energy per pulse required for the separate production of the antiprotons might be of the same order as that required for the conventional slow compression drive of the main fuel.

\section{Concept Description}

Antiprotons offer a unique way of packaging and delivering energy. With a releasable energy per unit mass of $\sim 180 \mathrm{MJ} / \mu \mathrm{g}$, they provide the highest specific energy of any known entity. The essence of antiproton fast ignition of an inertial confinement fusion (ICF) target is to deliver the fast ignition energy of a few kilojoules in the form of the rest energy of an antiproton cluster. Energy deposition then takes place at the center of the 
products. Similar to standard fast ignition [1], we desire high gain with only modest pre-compression of the main fuel and, therefore, seek to realize the same typical order of magnitude increase in gain or, equivalently, typical tenfold reduction in the pre-compression drive energy over conventional, hot-spot ICF.

In conventional, isobaric ignition of an ICF target, a single driver is employed to both compress and ignite the fuel $[2,3]$. Under such conditions, the assembled fuel is composed of a low density, high temperature central hot spot surrounded by a high density, low temperature main fuel region. The two regions are in pressure equilibrium because, during stagnation the hot spot has sufficient time to equilibrate with the cold compressed fuel surrounding it. By contrast, as expounded in the vanguard paper by Tabak et al. [1], the principle of "fast" ignition is to decouple the compression of a target from its ignition. First, a conventional slow ( 10's ns) driver is employed to compress the fuel. Then, providing the ignition energy can be delivered sufficiently rapidly by a separate fast $(\sim 10$ 's ps) system, the hot spot so created and the main fuel are not in pressure equilibrium. The isochoric (constant density) model of Kidder [4] can then be employed and much larger gains are predicted for the same fuel mass [1]. In the standard fast ignition concept of Ref. 1, the ignition energy is delivered by a low energy, high intensity, short pulse laser and transferred to the high density region by the transport of suprathermal electrons. By contrast, in antiproton fast ignition, the ignition "sparkplug" energy results from the annihilation of the antiprotons in the target and not from an outside driver.

Fig 1. shows two conceptual target capsules for this idea. In the first configuration - Scheme A in Fig 1(A) - the capsule is compressed by a conventional, slow compression system (e.g., through heavy ions and indirect drive). The antiprotons are then injected as a short pulse of ions, with the ion kinetic energy selected to deliver the antiprotons to the center of the imploded capsule where annihilation takes place. The kinetic energy of this beam pulse is small compared with the antiproton stored annihilation energy. A small, heavy metal inclusion of bismuth, lead, tungsten, or similar mass number is utilized in the target to enhance local energy deposition of the annihilation energy as described below. A fast ignition hot spot is then created at the center of the capsule and the thermonuclear burn front propagates radially outwards into the cold, dense main fuel region. Antiproton annihilation and energy deposition of the product particles take place in a time much shorter than the hydrodynamic disassembly time of the compressed fuel. Under such isochoric conditions, significantly more mass is compressed to much lower peak density, resulting in a gain and yield considerably larger that those achievable with conventional, isobaric ignition. 


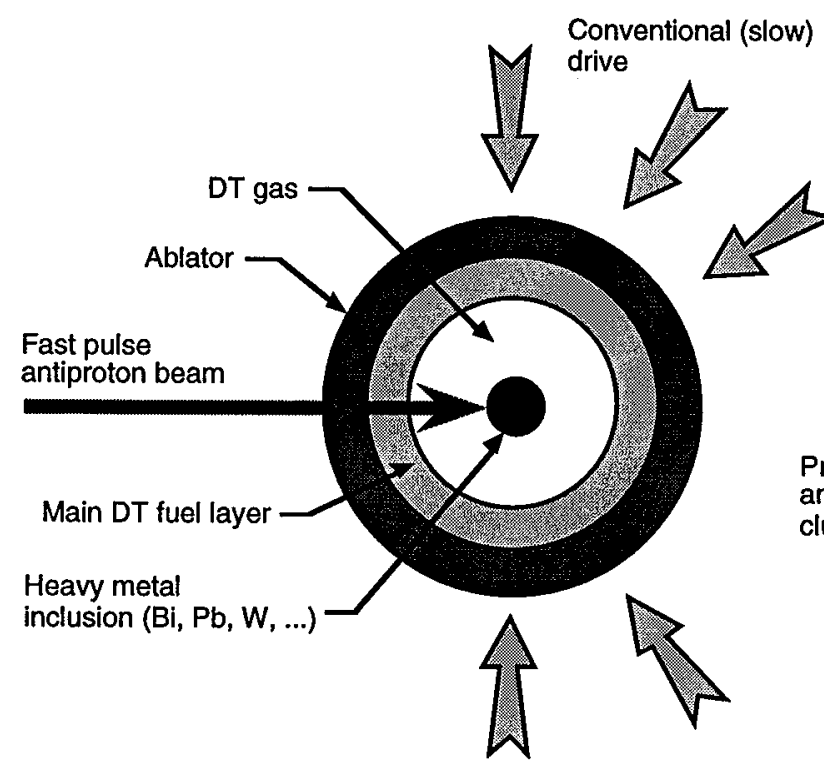

Scheme A: External Antiproton Injection

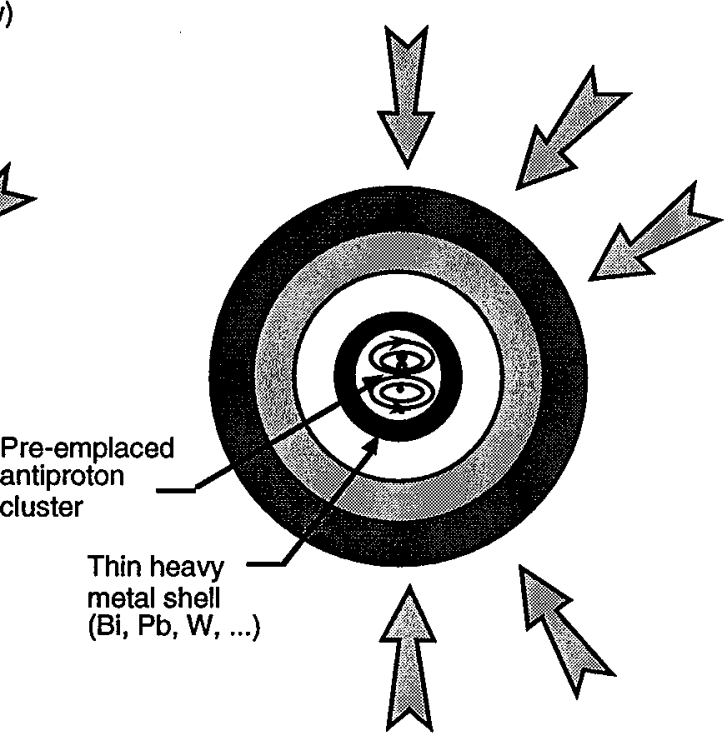

Scheme B: Autocatalytic Ignition

Fig. 1. Conceptual target capsules for antiproton fast ignition (not to scale)

In the second, "autocatalytic" concept for antiproton fast ignition Scheme B in Fig 1(B) - the antiproton package is pre-emplaced in ion form isolated by a magnetic field at the center of the fuel capsule before the main, slow compression occurs. This central, inner capsule is then squeezed down by the main drive to trigger the antiproton energy release. Again a heavy metal is included, this time in the form of a thin-walled shell, to enhance energy deposition of annihilation products.

In both Schemes $A$ and $B$, the fast ignition energy results from the deposition at the center of the target of a fraction of the annihilation energy. The antiprotons so employed would have been previously manufactured in a separate, optimized production system and stored until needed. Note also, that in the first scheme above, the kinetic energy of the injected antiproton beam necessary for central delivery is negligible compared with the fast ignition energy released through annihilation.

With such fast ignition schemes, appreciable gain may be realizable with as little as $100 \mathrm{~kJ}$ delivered by the main, slow compression drive and less than a tenth of this delivered by the fast ignition system. Typical fast ignitor gain predictions can be found in Ref. 1 where it is shown that, relative to conventional isobaric, hot spot ignition, an order of magnitude higher gain may be realizable for the same drive energy. Equivalently, for the same gain, an order of magnitude reduction of the drive energy may be realizable. 


\section{Features and Critical Issues}

The features of antiproton fast ignition relative to the standard fast ignition scenario employing a high intensity, short pulse laser include:

- Low injection energies ( Joules for the injected scheme in Scheme A; zero for autocatalytic scheme in Scheme B)

- Large energy delivery with a small package of antiprotons (antiproton stored energy is $180 \mathrm{MJ} / \mu \mathrm{g}$ )

- Central ignition, rather than edge ignition, either through control of external beam kinetic energy or by pre-emplacement

- Circumvents requirements for fast laser channeling ("hole boring") through halo corona, and transport of suprathermal electron to the high density core

- Conceivably could be utilized with indirectly-driven, heavy ion targets as laser channeling through houlrahm debris is not required

- Eliminates vulnerable final optics

- The second, autocatalytic scheme requires only a single, external drive system

Critical issues include:

- Very speculative. There is, as yet, no experience with manipulation of low energy antiprotons in analogous systems

- The injected antiproton scheme, Scheme A, requires precise focusing of a low kinetic energy proton beam with short pulse length. May require neutralized compression and focusing (using positrons) or plasma channel focusing.

- Heavy metal inclusions will complicate target fabrication and introduces problems of heavy metal mix.

- A separate antiproton production facility would be required. (Antiprotons can be produced and stored for long periods - months if required. We envisage such a facility as being part of a large, multiunit, inertial fusion energy reservation. The ultimate utility of such a fast ignition scheme will depend on the efficiency of antiproton manufacture in future, optimized production facilities) 


\section{Capsule Antiproton Energy Deposition}

Following the methodology of Ref. 1, the minimum energy for fast ignition, $E_{i}$ is that required to raise the temperature of the DT fuel to ignition temperature $T_{i}$ in a sphere of radius equal to the deposition range $(\rho r)_{\alpha}$ of a $3.5 \mathrm{MeV}$ fusion alpha particle. This enables the burn to propagate into the main cold fuel mass at the same compressed density. The required fast ignition energy $E_{i}$ then becomes

$$
E_{i}=N k\left(T_{i}+T_{e}\right) \approx \frac{8}{3} \pi r^{2}(\rho r)_{\alpha} k T_{i} / m_{D T}
$$

where $N$ is the number of DT fuel nuclei of average mass $m_{D T}$ at ignition temperature $T_{i}\left(\approx T_{e}\right)$ in the one-alpha-range sphere of radius $r$ and areal density $(\rho r)_{\alpha}$. We now determine how many antiprotons are required to yield a deposition energy in this sphere equal to $E_{i}$.

On entering a cold background plasma of electrons and ions, the initial kinetic energy of an antiproton beam is transferred along its path to the electrons of the medium through electron drag and the antiproton slows down similar to a conventional (positive), heavy charged particle. At an energy of a few 10's of eV, the (negative) antiproton will be captured by a ion in the medium and forms an "antiprotonic atom" which cascades towards the ground state by the emission of x-rays. Finally, the antiproton reaches an inner stable Bohr orbit of radius $m_{e} / m_{p}$ smaller than a conventional electron orbit. The antiproton then annihilates from this bound state with either a proton or neutron from within the nucleus it is bound to. The lifetime for this bound state is very short, i.e., $\ll<10^{-12}$ s. The total annihilation energy released is equal to $\sim 1.88 \mathrm{GeV}$, twice the rest mass of the antiproton.

It is also possible for the antiproton to undergo direct annihilation with a nucleus in flight at kinetic energies below a few tens of $\mathrm{MeV}$, although cross sections for this are small relative to electron drag and subsequent antiprotonic atom formation. Therefore, a beam of antiprotons annihilate at the end of their range and their stopping point can be precisely controlled by their initial kinetic energy.

Antiproton annihilation is a strong interaction process which takes place at the level of the quark structure of the nucleus. Annihilation of an antiproton with an isolated proton at rest is shown in Fig. 2, where we indicate typical average numbers of pions produced. The division of the $1.88 \mathrm{GeV}$ annihilation energy is, initially, $\sim 64 \%$ to the kinetic energy of the pions and $\sim 36 \%$ to their rest energy. In $\sim 5 \%$ of annihilation events, a kaon pair is also emitted. Because these meson products are all unstable, several 


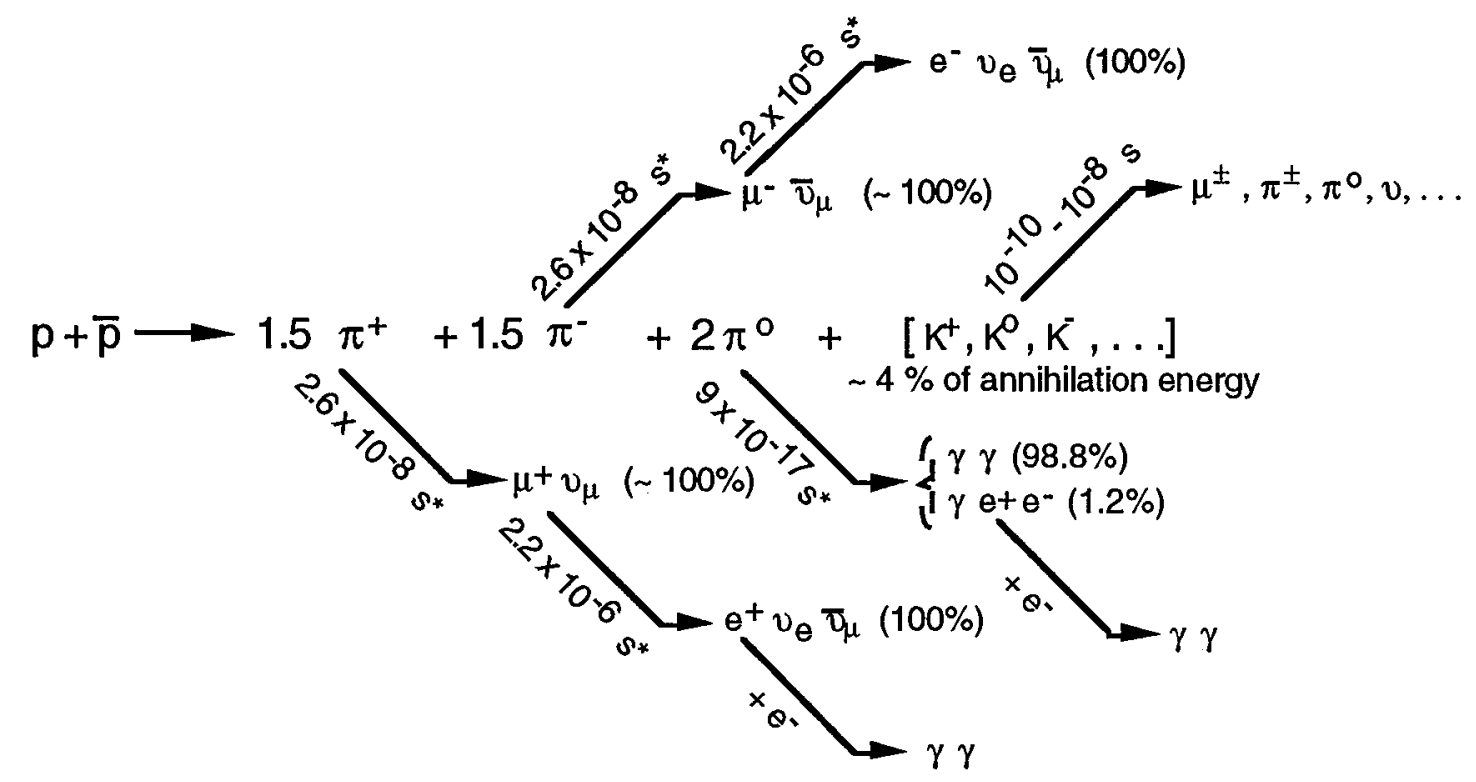

*Mean lifetime of particle $\left(t_{0}\right)$. In lab frame, lifetime is $\gamma t_{0}$ and is $\sim 7 \times 10^{-8} \mathrm{~s}$ for $\pi^{ \pm}$decay and $\sim 6 \times 10^{-6} \mathrm{~s}$ for $\mu^{ \pm}$decay.

Fig. 2. Typical products resulting from annihilation of an antiproton with a single isolated proton. Annihilation with a single isolated neutron is similar except for a change in the ratio of charged and neutral pions. Annihilation with a nucleon in a heavy nucleus results in nuclear breakup with significant energy transfer to the residual nuclear fragments (see Fig. 3)

decay chains subsequently occur as shown in Fig. 2. The neutral pion $\left(\pi^{\circ}\right)$ with its very short lifetime, travels less than a micron before decaying into two energetic gamma rays. Most of this gamma energy will escape the capsule and will require attention to shielding in the ICF reactor chamber. The charged pions $\left(\pi^{ \pm}\right)$decay into muons $\left(\mu^{ \pm}\right)$and neutrinos $\left(v_{e}, m\right)$. The muons then further decay into electrons $\left(\mathrm{e}^{-}\right)$and positrons $\left(\mathrm{e}^{+}\right)$, and the latter subsequently annihilate with electrons in the surrounding medium. Reaction products resulting from antiproton annihilation with an isolated proton are slightly different from those from annihilation with an isolated neutron*. In the latter, the mean number of negative pions is one greater than the mean

\footnotetext{
* The proton is composed of two "up" quarks, each of charge $+2 / 3$, plus one "down" quark of charge $-1 / 3$. The neutron is composed of two "down" quarks plus one "up" quark, while the antiproton has two "anti-up" quarks (charge -2/3) plus one "anti-down" quark (charge $+1 / 3$ ). The mesons resulting from the annihilation reactions are composed of quark-antiquark pairs; e.g. the positive pion is composed of an "up" quark (charge $+2 / 3$ ) and an "anti-down" quark (charge $+1 / 3$ ).
} 
number of positive pions and the ratio of charged to neutral pions is somewhat greater [5].

The above discussion pertains to annihilation of an antiproton with a single, isolated proton (i.e., hydrogen nucleus) or neutron. Annihilation with the protons or neutrons within in a heavy nucleus of $A \gg>1$ produces rather different products and energetic. An appreciable fraction of available annihilation energy is transferred to the nucleus via pion interactions as shown schematically in Fig. 3. The nucleus breaks up resulting in highly ionizing, short range nuclear fragments.

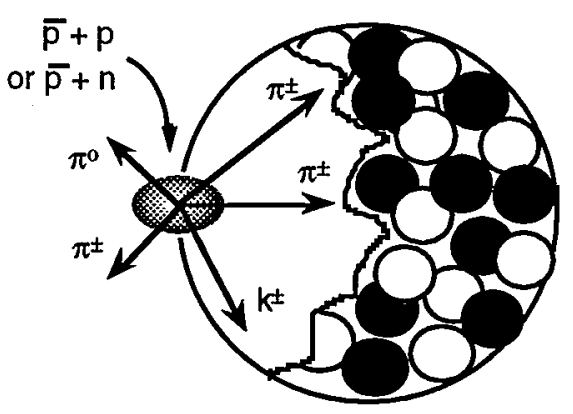

Antiproton annihilates with a proton or neutron on the surface of the heavy nucleus. A fraction of the annihilation products are directed inwards and interact within the high density nuclear material

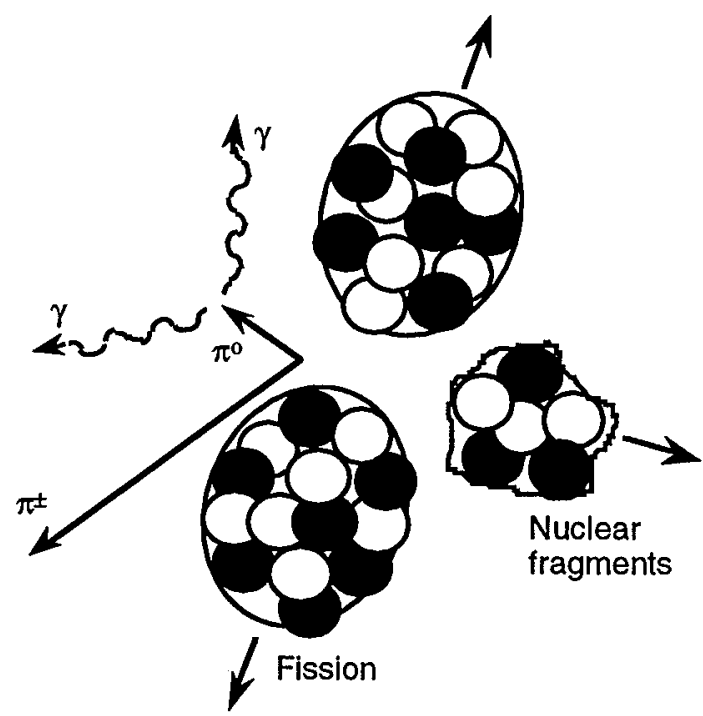

The inward-directed products cause nuclear breakup resulting in highly ionizing, short range fragments. The outward-directed primary products and daughters have long ranges and deposit the majority of their energy outside the ignitor region

Fig. 3. Annihilation of an antiproton in a heavy nucleus takes place with a neutron or proton at the nuclear surface. This results in nuclear breakup with significant energy transfer to the residual nuclear fragments.

In general, therefore, not all of the total $1.88 \mathrm{GeV}$ of annihilation energy will be deposited in the fast ignitor zone. We take advantage of the fact that annihilation in a compound nucleus results in transfer of annihilation energy to nuclear fragments by employing a heavy metal inclusion such as bismuth, lead, tungsten, thallium, mercury, etc., in the form of a ball or shell as shown, respectively, in Fig. 1(A) and (B). Also, because the critical energy to promote fission in such heavy nuclei is only a few $\mathrm{MeV}$ to 10 's of $\mathrm{MeV}$, 
fission will occur when antiprotons annihilate in such nuclei ${ }^{*}$. For example, annihilation with uranium has shown to induce fission in $\sim 100 \%$ of the events [6]. Because such fission energy appears as highly ionizing fragments, all this additional energy will be deposited in the ignitor zone.

As shown in Fig. 3, antiproton annihilation with a heavy nucleus specifically takes place with a proton or neutron on the surface of the nucleus. The outward directed pions or kaons have long ranges even in the precompressed fuel - for example, $\sim 30 \mathrm{~g} / \mathrm{cm}^{2}$ for $100 \mathrm{MeV}$ pions [7] so only a small fraction of their ionization energy is deposited in the ignitor zone. Similarly, the neutral pion decays in $\sim 10^{-18} \mathrm{~s}$ into two energetic gamma rays which also leave the ignitor zone.

By contrast, the inward directed mesons encounter a medium of nuclear density. Their interaction mean free path is then

$$
\lambda=[n \sigma]^{-1} \approx \frac{\frac{4}{3} \pi r_{N}^{3}}{A \sigma}
$$

where $n$ is the nucleon density, $\sigma$ is the pion-nucleon interaction cross section, $A$ is the nuclear mass number and $r_{N}$ is the nuclear radius. Taking $\sigma \sim 100 \mathrm{mb}$ for $\sim 100 \mathrm{MeV}$ pions [8] and $r_{N}=1.2 A^{1 / 3} \times 10^{-15} \mathrm{~m}$ gives a nuclear density of $\sim 1.4 \times 10^{44} \mathrm{~m}^{-3}$ for $\mathrm{A} \sim 230$ and, therefore, a pion mean free path of only $\sim 7 \times 10^{-16} \mathrm{~m}$. This is about a tenth of a nuclear radius thus ensuring that virtually all the inward directed pion energy is transferred to the nucleus and

\footnotetext{
* In conventional, neutron-induced fission, a neutron is added to a target nucleus of mass number A. Fission of the A+1 nucleus occurs when the kinetic energy of the incident neutron plus its binding energy is greater than the critical energy, $E_{c r i t}$, required for this $A+1$ nucleus to penetrate its internal Coulomb barrier so that the two fission fragments can separate. In fissile nuclei (e.g., $235 \mathrm{U})$, the binding energy $\mathrm{E}_{\mathrm{b}}$ released by the neutron in forming the compound $\mathrm{A}+1$ nucleus (e.g., ${ }^{236} \mathrm{U}$ ) is greater than $\mathrm{E}_{\text {crit }}$ so the compound nucleus can fission with zero neutron kinetic energy and such target nuclei have high thermal fission cross sections. In other nuclei (e.g., ${ }^{238} \mathrm{U},{ }^{232} \mathrm{Th}$ ), $\mathrm{E}_{\mathrm{b}}$ is slightly less than $\mathrm{E}_{\mathrm{crit}}$ by an $\mathrm{MeV}$ or so and the balance must be supplied by the kinetic energy of the incident neutron. An example of this is $238 \mathrm{U}$ which undergoes only fast fission, with a threshold of $\sim 1 \mathrm{MeV}$ required for the incident neutron. For heavy nuclides in the range ${ }^{233} \mathrm{Bi}$ down to ${ }^{184} \mathrm{~W}$, using a formalism from Foderaro [9], we estimate that critical energies $E_{c r i t}$ of a minimum of $\sim 8 \mathrm{MeV}$ to several 10 's of $\mathrm{MeV}$, respectively, must be supplied to promote fission, otherwise deexcitation by gamma emission is the preferred route. Such critical energies are supplied by the inward directed pion products with the excess going to the kinetic energy of the nuclear fragments.
} 
its subsequent breakup. The resulting nuclear products are highly ionizing with short ranges.

Therefore, the net annihilation energy deposition in the ignitor zone per antiproton is:

$\begin{aligned} E_{A, n e t}= & \begin{array}{l}\text { Energy deposited in } \\ \text { the ignitor zone by } \\ \text { outward directed } \\ \text { annihilation } \\ \text { products }\end{array} \quad \begin{array}{l}\text { Energy deposited } \\ \text { in the ignitor } \\ \text { zone by nuclear }\end{array}\end{aligned} \quad \begin{aligned} & \text { Energy deposited } \\ & \text { fragments }\end{aligned}$

That is

$$
E_{A, \text { net }} \approx E_{A}\left(I-f_{i}\right) \eta_{A}+E_{A} f_{i} f_{N} \eta_{N}+E_{f} N_{f} \eta_{f}
$$

where,

$$
\begin{aligned}
& E_{A}= \mathrm{p}-\overline{\mathrm{p}} \text { annihilation energy (i.e. twice the antiproton rest energy, } \\
&1.88 \mathrm{GeV}) \\
& f_{i}= \begin{array}{l}
\text { fraction of annihilation pion products created at the nuclear } \\
\text { surface which are inward directed }
\end{array} \\
& \eta_{A}=\begin{array}{l}
\text { energy deposition fraction of outward directed pions products } \\
\text { within the ignitor zone }
\end{array} \\
& f_{N}=\begin{array}{l}
\text { energy deposition fraction of inward directed pions products } \\
\text { within the nucleus }
\end{array} \\
& \eta_{N}=\begin{array}{l}
\text { energy deposition fraction of nuclear fragments within the ignitor } \\
\text { zone. }
\end{array} \\
& E_{f}=\text { energy released per heavy metal fission } \\
& N_{f}=\text { number of fissions per antiproton annihilation (may be greater } \\
& \eta_{f}=\text { than unity from fission neutron propagation) }
\end{aligned}
$$

For an estimation of the various energy deposition fractions of charged products within the ignitor zone, we use

$$
\begin{aligned}
\eta_{X} & \approx(\rho r)_{\alpha} /(\rho r)_{X} & \text { for }(\rho r)_{X}>(\rho r)_{\alpha} \\
& \approx 1 & \text { for }(\rho r)_{X} \leq(\rho r)_{\alpha}
\end{aligned}
$$

where $(\rho r)_{\alpha}$ is the areal density of our unity alpha range sphere and $(\rho r)_{x}$ is the range of various products particles above. From the arguments above, we would expect that, due to the range of energetic pions in compressed fuel, $\eta_{A}$ would be small, whereas pion energy transfer to the nucleus and subsequent breakup into high charge state, highly ionizing products (see Eq. 2) would 
yield values of $f_{N^{\prime}} \eta_{N}$ and $\eta_{f}$ close to unity. Also, because of the long range of fission neutrons, only the primary fission is likely to occur in the ignitor zone, i.e., $N_{f} \sim 1$

The number of antiprotons required per ICF capsule is then

$$
N_{\bar{p}}=\frac{E_{i}}{E_{A, n e t}}
$$

Note also from Fig. 2, that negative muons are produced in the decay of the annihilation pions. Re-deposition of these muons in the DT fuel could, in principle, promote further fusions by muon catalysis. Here, the heavy negative muon binds tightly to a fuel nucleus and reduces the width of the conventional Coulomb barrier between it and a neighboring fuel by a factor of $m_{\mu} / m_{e} \sim 200$, thus permitting fusion at $\mathrm{eV}$ temperatures and below [10]. Typically, a maximum of 150 DT fusions can be catalyzed per muon where the latter is ultimately lost by sticking to an outgoing alpha particle. This could, in principle, result in an additional alpha deposition of up to $\sim 500 \mathrm{MeV}$ per original antiproton (see below). However, only a small fraction of the muons so produced will be captured in the fuel. In general, the lifetime for $\pi^{-}$ decay to muons is $\sim 70 \mathrm{~ns}$ in the lab frame which is too slow for muon catalysis to be counted in the initial fast ignition process. Furthermore, subsequent muon decay ( $\sim 6 \mu \mathrm{s}$ in the lab frame) is too fast to permit muon collection and re-injection into a subsequent capsule unless the latter is a separate component of the same target assembly. Accordingly, we will not assess muon catalysis in the energy deposition process.

\section{Antiproton Production}

Antiprotons are routinely produced today at several of the world's large proton synchrotrons. They are employed as engineering "tools" in particle physics research by accelerating them to high energies in protonantiproton colliders. Initial production takes place by directing accelerated protons onto a cooled target, usually of tungsten, according to the reaction:

$$
p+p \rightarrow p+p+p+\bar{p}
$$

This minimum set of product particles is necessary for baryon conservation and the threshold energy required is $1.88 \mathrm{GeV}$ in the center-of-mass or $5.63 \mathrm{GeV}$ for a stationary target. Typically energies somewhat greater than threshold are required for adequate yield. The antiprotons so formed are directed by a magnetic lens and, following cooling to reduce energy spread, are then decelerated to several tens to hundreds of $\mathrm{MeV}$ within a collecting/storage ring. 
At CERN, for example, the ACOL antiproton production facility can produce $>10^{12} \overline{\mathrm{p}} /$ day, and the Low Energy Antiproton Ring (LEAR), stores antiprotons at $\sim 200 \mathrm{MeV}$ with a final energy spread of $<<3 \%$. Note that the goals of the world's high energy physics programs are typically satisfied with a modest production of only $\sim 10^{15} \overline{\mathrm{p}} /$ year.

The overall production efficiency of antiprotons on these far from optimized systems is rather low, i.e., $\sim 10^{-4}$. Speculation of ultimate efficiencies for optimized antiproton production in colliding beam facilities are given in Ref. 11.

The wallplug energy required to produce the $N_{\bar{p}}$ antiprotons required in Eq. 5 above is then

$$
E_{\bar{p}, w}=N_{\bar{p}} E_{a c} /\left(\eta_{a c} \eta_{s}\right)
$$

where,

$E_{a c}=$ kinetic energy of the production accelerator proton beam

$\eta_{a c}=$ energy efficiency of the accelerator (wallplug to accelerated beam)

$\eta_{s}=$ efficiency of the antiproton production, collection and storage

\section{Antiproton Delivery and Containment}

In the antiproton injection scheme, i.e. Scheme $A$ in Fig 1(A), the kinetic energy, $E_{k}$ of the beam particles need only be sufficient for penetration to the required annihilation point. The total beam energy per pulse is then

$$
E_{\text {beam }}=N_{\bar{p}} E_{k}
$$

Since a kinetic energy of only $\sim 10 \mathrm{MeV}$ is required for protons to penetrate a pr 1 [7], the total energy in the beam pulse will be typically two orders of magnitude smaller than the fast ignition energy and, moreover, is distributed throughout the slowing down path of the antiprotons. Therefore, this energy contributes negligibly to the capsule fast ignitor energetics.

The beam current required is

$$
I_{\text {beam }}=N_{\bar{p}} e / \tau_{i}
$$

where $\tau_{i}$ is the fast ignition pulse time. 
Although this would result in a low current requirement relative to those from typical pulsed light ion diodes, the low beam energy will result in a high perveance, $K \sim I / V$, which will make it difficult to focus the beam to the small spot size required without neutralized pulsed compression and neutralized focusing. Note that neutralizing an antiproton beam would necessitate adding positrons, not electrons. An alternative might be to employ plasma channel focusing through a low density, conventional (normal matter) plasma background.

In the case of the autocatalytic scheme - Scheme B in Fig. 1(B) antiprotons are confined in the center of the capsule. The capsule is subsequently imploded by the slow compression system which is the only external drive then required. At small compression radii, the confinement of the antiprotons is destroyed and the antiprotons annihilate with the compressed, thin-walled heavy metal shell yielding similar deposition characteristics as above. In ion form, the antiprotons would require a preemplaced magnetic confining field at the center of the capsule. Methods to create such magnetic fields in small capsules have been considered [12, 13, 14] although presumably this would complicate target fabrication. Also, sustainment of this field via external connections may perturb capsule implosion symmetry.

The limit on the density of antiprotons stored as a nonneutral, single ion plasma in a confining field is set by the Brillouin limit expressing the space charge equilibrium condition. This requires

$$
\frac{N_{\bar{p}} m_{p} c^{2} / \frac{4}{3} \pi r_{s}^{3}}{B^{2} / 2 \mu_{o}} \leq 1
$$

where $r_{s}$ is the initial, uncompressed radius of the thin heavy metal shell. That is, the applied axial magnetic field containing the-radial electric space charge of the non neutral plasma must have an energy density greater than the rest mass energy density of the ions so contained. Typically, only a few percent of this ideal limit has been obtained experimentally to date. Thus the large fields necessary ( hundreds of Teslas - see below) probably make this non-neutral, single ion storage impractical.

In the case of pre-emplacement as a neutral plasma of antiprotons and positrons, the storage limit and required magnetic field would be determined by the macroscopic beta (pressure) limit, i.e.

$$
\beta=\frac{2 N_{\bar{p}} k T / \frac{4}{3} \pi r_{s}^{3}}{B^{2} / 2 \mu_{o}} \leq 1
$$


Employment of a field-reversed configuration, might permit beta limits close to unity [13]. However, without sustainment of the magnetic field - either by an external supply or superconducting materials - field diffusion would necessitate rapid target assembly before compression.

Interestingly, from Eqs. 9 and 10 above, the ratio of the magnetic field required to contain the antiprotons as a neutral plasma to that required to contain the equivalent as a nonneutral plasma is

$$
\frac{B_{\text {neutral }}}{B_{\text {nonneurral }}} \approx \sqrt{\frac{2 k T}{m_{p} c^{2}}}
$$

which, for an initial ion temperature of, say, $10 \mathrm{eV}$, is $\sim 10^{-4}$, thus underlining the value of neutralization.

To date, antiprotons have been manipulated and stored mainly in large storage rings. Low energy protons have also been manipulated and transported in portable Penning Traps [15].

There have been suggestions of antiproton storage in neutral matter form by first creating antihydrogen by addition of positrons, followed by the formation of anti-molecular-hydrogen [11]. Such neutral antimatter would, of course, have to be isolated from normal matter. A recent experiment at CERN [16] has created antihydrogen atoms by traversing a xenon jet with an antiproton beam. Here, a small fraction of the antiprotons convert their energy to electron-positron pairs and a fraction of subsequent antiprotons pick up the positrons. A future proposed CERN experiment will collect cooled antiprotons and positrons in a separate Penning traps at milli-eV temperatures (i.e., a few hundred Kelvin). These will then be bled into a third trap to form antihydrogen [17].

One method to manipulate bulk antimatter might be in the form of cluster ions, $\mathrm{H}_{\mathbf{n}}{ }^{-}$, i.e. an ion of $\mathrm{n}$ antihydrogen atoms with a positron removed. According to Stwalley [18], formation of anti- $\mathrm{H}$, anti- $\mathrm{H}^{+}$and anti$\mathrm{H}_{2}$ - could proceed by a number of methods. Molecular antihydrogen, i.e. anti$\mathrm{H}_{2}$, would be the most difficult of the simple species to form and especially to manipulate because of small magnetic moments and complex vibrational states. However, both the ionic species $\mathrm{H}_{2}^{-}$and $\mathrm{H}_{2}{ }^{+}$would be stable if formed and, in principle, would be more easily controlled and manipulated via magnetic, electrostatic and RF techniques. However, it would appear difficult to perform such manipulations within an ICF capsule. Antihydrogen ice formation via inverse sublimation would require methods for latent heat removal and nucleation that do not involve a wall. Levitation of antihydrogen ice would depends on its form -- orthohydrogen (spins parallel) 
or parahydrogen (spins antiparallel). The latter is diamagnetic and might be stably levitated in a static field as has been demonstrated with graphite of similar diamagnetic susceptibility [19]. Active electrostatic levitation with feedback position control might also be performed and would require charging of the ice particles by, say, irradiation with UV light. An analogous trap has been constructed at JPL and has levitated a $20 \mathrm{mg}$ ball of $\mathrm{H}_{2} \mathrm{O}$-ice in the earth's gravitational field [20].

\section{Example Case}

Here we provide some order of magnitude estimations for the parameters defined above applied to an example case. The key results are summarized in Table 1.

We assume cold pre-compression of the fuel by a slow (10's of nsec) compression system e.g. a heavy ion driver. We take a fast ignition requirement characterized by an ignition energy $E_{i}=5 \mathrm{~kJ}$, an areal density for $3.5 \mathrm{MeV}$ alpha deposition of $(\rho r)_{\alpha}=0.3 \mathrm{~g} / \mathrm{cm}^{2}$ and ignition temperature of $T_{i}=7 \mathrm{keV}$. Under these conditions, the burn will propagate into the cold fuel mass. From Eq. 1, the hot spot radius is then $r \sim 30 \mu \mathrm{m}$. We require this to occur in a time, $\tau_{i}$, short relative to the disassembly time, i.e. a time of $\tau_{i,} \sim r / c_{s} \approx 40 \mathrm{ps}$ where $c_{s}$ is the sound speed.

From Eqs. 3-5, with $E_{A}=1.88 \mathrm{GeV}, E_{f} \sim 200 \mathrm{MeV}$, and taking $f_{i} \sim 0.4$, $\eta_{A} \approx(\rho r)_{\alpha} /(\rho r)_{\pi} \sim 0.3 / 30=0.006, f_{N}=\eta_{N}=\eta_{f} \approx 1$ and $N_{f} \sim 1$, the number of antiprotons required to realize this deposition energy in the fast ignitor zone is $N_{\bar{p}} \sim 3 \times 10^{13}$. Note that under these assumptions, fission comprises some $20 \%$ of the $5 \mathrm{~kJ}$ deposition energy in the ignitor zone.

Note that we did not include the muons resulting from the decay of the negative pions (see Fig 2.) in the above accounting because of the relatively long time scales involved and because we would expect the majority to be produced in flight, outside the ignitor zone. However, if in some way they could be captured, we might achieve a further $\sim 1.5 \times 150 \times 3.5 \mathrm{MeV}=800 \mathrm{MeV}$ of alpha particle deposition energy per antiproton annihilated, through muon catalysis. The factor of 1.5 is the production ratio of $\mu^{-}$(through $\pi^{-}$decay) per $\mathrm{p}-\overline{\mathrm{p}}$ annihilation. Also, we assume a chain length of 150 DT fusions before muon loss due to alpha capture which is the present experimentally observed value. Note that, fortuitously, this would, if harnessed, yield a total of $\sim 4 \mathrm{~kJ}$ of alpha deposition energy per target, similar to the magnitude of the fast ignition energy. Again, however, due to the disparity of the timescales for pion and muon decay discussed above, we do not include this in the fast ignition energy accounting. 
Table 1. Results for Sample Case

\begin{tabular}{|c|c|c|}
\hline & $\begin{array}{c}\text { Scheme A } \\
\text { Injected } \\
\text { Antiproton Fast } \\
\text { Ignition }\end{array}$ & $\begin{array}{c}\text { Scheme B } \\
\text { Autocatalytic } \\
\text { Antiproton Fast } \\
\text { Ignition }\end{array}$ \\
\hline Fast ignition deposition energy $(\mathrm{kJ})$ & $5 \mathrm{~kJ}$ & $\leftarrow$ \\
\hline Required no. of antiprotons per target & $\sim 3 \times 10^{13}$ & $\leftarrow$ \\
\hline Capsule gain & $\sim 1000$ & $\leftarrow$ \\
\hline $\begin{array}{l}\text { Main, slow drive energy (e.g. heavy } \\
\text { ions) }\end{array}$ & $\sim 3 \mathrm{MJ}$ & $\leftarrow$ \\
\hline $\begin{array}{l}\text { Wallplug energy per target for main } \\
\text { drive }\end{array}$ & $\sim 10 \mathrm{MJ}(\mathrm{e})$ & $\leftarrow$ \\
\hline $\begin{array}{l}\text { Wallplug energy per target for } \\
\text { antiproton production }\end{array}$ & $\sim 5 \mathrm{MJ}(\mathrm{e})$ & $\leftarrow$ \\
\hline Antiproton injection energy per pulse & $\sim 48 \mathrm{~J}$ & N.A \\
\hline Injection kinetic energy per particle & $\sim 10 \mathrm{MeV}$ & N.A \\
\hline Injection current per pulse & $\sim 160 \mathrm{kA}$ & N.A \\
\hline Injection time & $\sim 30 \mathrm{ps}$ & $\begin{array}{l}<30 \mathrm{ps}(\overline{\mathrm{p}} \text { reaction } \\
\text { time) }\end{array}$ \\
\hline Capsule confining field & N.A & $\sim 0.06 \mathrm{~T}$ \\
\hline Capsule antiproton number density & N.A & $7 \times 10^{20} \mathrm{~m}^{-3}$ \\
\hline
\end{tabular}


If we assume that the requirements of the main slow drive - say, heavy ions - are similar to that for the standard fast ignitor concept [1], then, somewhat conservatively, a capsule gain of $\sim 1000$ may be realizable for a few megajoules of heavy ion energy into the external houlrahm. With a heavy ion driver efficiency of $\sim 30 \%$, this results in a wallplug electrical energy of $\sim 10 \mathrm{MJ}(\mathrm{e})$ for the main driver.

We now turn to the electrical energy required to manufacture the antiprotons in a separate production facility external to the ICF reactor system. From Eq. 6 with $E_{a c} \sim 10 \mathrm{GeV}$ and taking an extrapolation of $\eta_{a c} \eta_{s} \sim 0.01$ for a future, optimized center-of-mass production system [11], yields an original investment in wallplug energy to produce the antiprotons of $\sim 5 \mathrm{MJ}(\mathrm{e})$ of electrical energy per target; that is, about the same order of magnitude as the main driver electrical wallplug requirements. Clearly, attention to the efficiency of production and collection in an ultimate production system is a critical issue in the feasibility of such as scheme.

For the injected antiproton fast ignition system, Scheme A in Fig. 1(A), the major energy is invested in making the antiprotons and not in their kinetic energy of injection. From Eqs. 7 and 8, assuming a beam kinetic energy per particle of $E_{k}=10 \mathrm{MeV}$ to penetrate a $\rho r$ of $\sim 1 \mathrm{~g} / \mathrm{cm}^{2}$ and fast ignition time of $\tau_{i} \sim 30 \mathrm{ps}$, yields a total beam energy per pulse of $E_{\text {beam }} \sim 48 \mathrm{~J}$ and a beam current of $I_{\text {beam }} \sim 160 \mathrm{kA}$. The perveance of such a beam would be $\sim 2.9$ probably necessitating neutralized pulse compression and focusing to achieve spot sizes $<<1 \mathrm{~mm}$.

For the autocatalytic scheme in Fig 1 (B), storage of the $N_{\bar{p}} \sim 3 \times 10^{13}$ antiprotons as a nonneutral, single ion species inside a thin heavy metal shell with an initial inner radius of, say, $r_{s} \sim 2 \mathrm{~mm}$ would, from Eq. 9., require a confining field of $\sim 500 \mathrm{~T}$ for a Brillouin limit of unity. This is impractical and, given that the un-neutralized ion density is $\sim 7 \times 10^{20} \mathrm{~m}^{-3}$, unsurprising. From Eq. 10, storage as a low temperature neutral plasma in say an FRC configuration at unity beta would require a magnetic field of only $\sim 0.03-0.09 \mathrm{~T}$ for initial ion temperatures in the range $1-10 \mathrm{eV}$.

Finally, we note that the benefit of the fission contribution to energy deposition from the heavy metal could be considered problematic not only because of mix contamination of the fuel but also because of the resulting residue of fission products. However, relative to a conventional fission reactor of the same thermal output as our ICF power plant, the production rate of such radionuclides would be reduced by a ratio $r$ of order

$$
r \approx f_{f} E_{i} /\left(G E_{d}\right)
$$


where,

$$
\begin{aligned}
& f_{f}=\text { fraction of the fast ignition deposition energy due to fission } \\
& G=\text { capsule gain } \\
& E_{d}=\text { main drive energy }
\end{aligned}
$$

Taking, from above, $G \sim 1000, E_{d} \sim 3 \mathrm{MJ}$ and $f_{f} \sim 20 \%$, yields a radionuclide ratio relative to fission of $\sim 3 \times 10^{-7}$. Interestingly, this is about the same potential reduction in biological hazard potential envisaged for magnetic confinement fusion relative to fission, if advanced material such as $\mathrm{SiC}$ or $\mathrm{V}$-alloys were used to mitigate the problems of neutron-activation in the first wall and blanket [21].

\section{Acknowledgments}

The author is pleased to acknowledge informative discussions with M. Tabak, J. H. Hammer, B. G. Logan, E. Lee, D. D. Ryutov, J. J. Barnard and T. Thomson.

\section{References}

1. M. TABAK, J. HAMMER, W. GLINSKY, W. KRUER, et al., Phys. Plasmas, 11626 (1994)

2. J. MEYER-TER-VEHN, Nucl Fusion, 22, 561 (1982)

3. V. B. ROZANOV, C. P. VERDON, M. DECROISETTE, S. YU, et al., "Inertial Confinement Target Physics", Chapter 2 in Energy from Inertial. Fusion, W. J. Hogan (Ed), International Atomic Energy Agency, Vienna, STI/PUB/944 (1995)

4. R. E. KIDDER, Nucl Fusion, 16, 405 (1976)

5. D.L. MORGAN in Proc Conf. on Annihilation in Gases and Galaxies, Goddard Space Flight Center, Greenbelt, MD, July 1989..

6. G.A. SMITH, p. 296 in Proc. of the RAND Workshop on Antiproton Science and Technology, October 6-9, 1987; B.W. Augerstein et al., (Eds.) (World Scientific, Singapore, 1988).

7. M. AGUILAR-BENITREZ et al (Eds), "Particle Properties Booklet", American Institute of Physics (1992) 
8. H. SCHOPPER (Ed), "Total Cross Sections for Reactions of High Energy Particles", New Series, Vol 12a-b, (Landolt-Bornstein, 1987)

9. A. FODERARO, "The Elements of Neutron Interaction Theory", p486ff (MIT Press, 1971)

10. S. E. JONES, Phys. Rev. Lett. 51, 1757 (1983)

11. R.L. FORWARD, "Antiproton Annihilation Propulsion - Final Report" US Air Force Rocket Propulsion Laboratory AFRPL TR-85-034/055 (1985)

12. N.MATTOR, J. H. HAMMER, “Magnetically Ignited Inertial Confinement Fusion", Bull. Amer. Phys. Soc. 40, 1781 (1995)

13. R. P. DRAKE, J. H. HAMMER, C. W. HARTMAN, L. J. PERKINS, D.D. RYUTOV, Fusion Technology 30, 310 (1996)

14. L. J. PERKINS, R. P. DRAKE, J. L. EDDLEMAN, J. H. HAMMER et al., "High Density, High Magnetic Field Concepts for Compact Fusion Reactors", 16th IAEA Fusion Energy Conference, Montreal, Canada, October 1996, IAEA-F1-CN-64/GP-18, (1997)

15. G. GABRIELSE, Phys. Rev. Lett., 272504 (1986).

16 G. BAUR, et al., Phys Lett, B368, 251 (1996)

17. J. EADES, Nature 379, 674 (1996)

18. W.C. STWALLEY, p. 373 and 393 in Proc. of the RAND Workshop on Antiproton Science and Technology, October 6-9, 1987; B.W. Augerstein et al., (Eds.) (World Scientific, Singapore, 1988).

19. R. WALDRON, Rev. Sci. Instr., 3729 (1966).

20. W. RHIM., "Development of an Electrostatic Levitator at JPL," p. 115 in Material Processing in the Reduced Gravity Environment of Space, G. Rindone (Ed.), (Elsevier 1982).

21. R.W. CONN, J. P. HOLDREN, S. SHARAFAT, D. STEINER et al., Nucl Fusion, 30, 1919 (1990) 